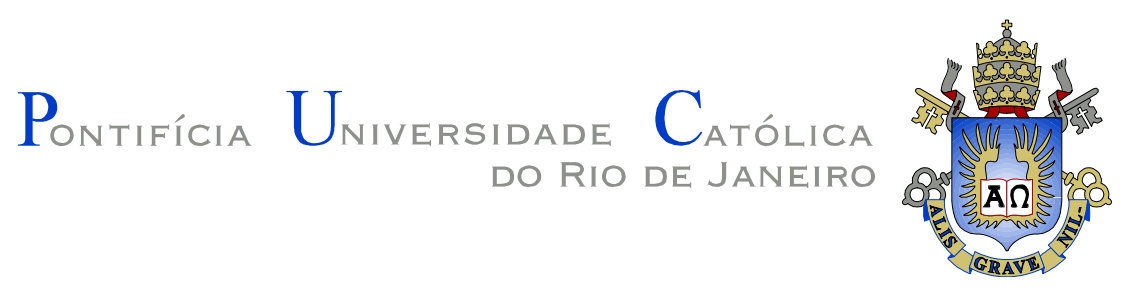

Luiz Miguel Régula

\title{
Padrões Virtuais e Tolerâncias Colorimétricas no Controle Instrumental das Cores
}

Dissertação de Mestrado

Dissertação apresentada ao Programa de Pós-Graduação em Metrologia da PUC-Rio como requisito parcial para obtenção do título de Mestre em Metrologia.

Orientador: Prof. Robert Hirschler Co-orientador: Prof. Maurício Nogueira Frota 
Luiz Miguel Régula

\title{
Padrões Virtuais e Tolerâncias Colorimétricas no Controle Instrumental das Cores
}

\begin{abstract}
Dissertação apresentada como requisito parcial para obtenção do grau de Mestre pelo Programa de Pós-Graduação em Metrologia do Centro Técnico Científico da PUC-Rio. Aprovada pela Comissão Examinadora abaixo assinada.
\end{abstract}

\author{
Prof. Robert Hirschler \\ Orientador \\ Centro de Tecnologia da Indústria Química e Têxtil \\ SENAI/CETIQT
}

Prof ${ }^{\mathrm{a}}$. Elisabeth Costa Monteiro Programa de Pós-Graduação em Metrologia Pontifícia Universidade Católica do Rio de Janeiro - PUC-Rio

Prof ${ }^{a}$. Paula Medeiros Proença de Gouvea

Programa de Pós-Graduação em Metrologia Pontifícia Universidade Católica do Rio de Janeiro - PUC-Rio

\section{Profa ${ }^{a}$. Danays Morejón González} Departamento de Física Pontifícia Universidade Católica do Rio de Janeiro - PUC-Rio

Prof. José Eugenio Leal

Coordenador Setorial do Centro Técnico Científico - PUC-Rio

Rio de Janeiro, 22 de março de 2004 
Todos os direitos reservados. É proibida a reprodução total ou parcial do trabalho sem autorização da universidade, do autor e do orientador.

\section{Luiz Miguel Régula}

Graduou-se em 1978 em Engenharia Operacional Mecânica pela Escola Naval. Pós-Graduado em 1988 em Pesquisa Operacional pela Universidade Federal Fluminense. Participou de diversos congressos promovidos pela Sociedade Brasileira de Pesquisa Operacional (SOBRAPO) e de Encontros Nacionais de Engenharia de Produção (ENEGEP). Participou de vários cursos na área de Metrologia e de Sistemas da Qualidade. Trabalha atualmente na área de Avaliação Operacional de Sistemas, no Centro de Análises de Sistemas Navais.

Ficha catalográfica

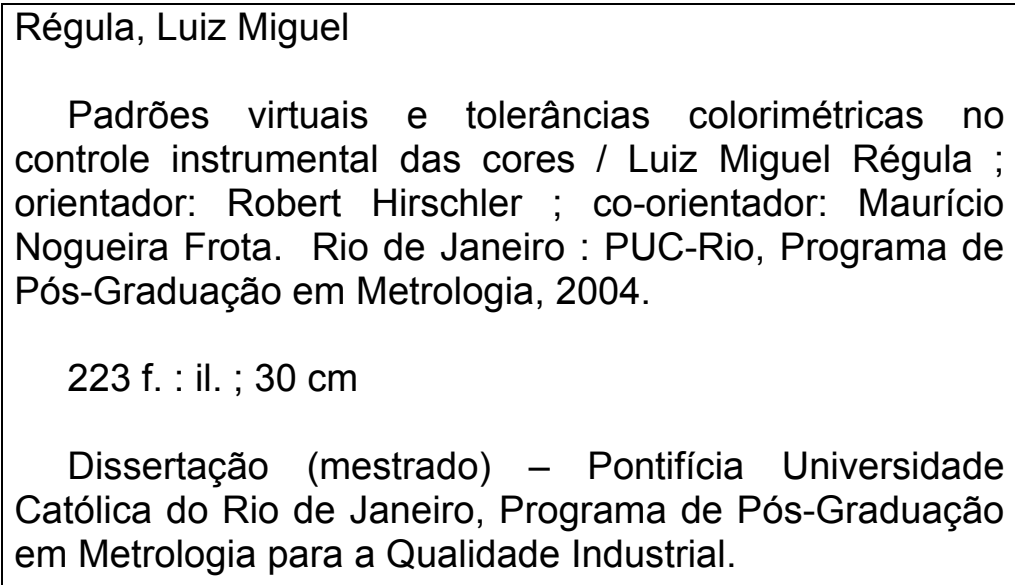

Padrões virtuais e tolerâncias colorimétricas no controle instrumental das cores / Luiz Miguel Régula ; orientador: Robert Hirschler ; co-orientador: Maurício Nogueira Frota. Rio de Janeiro : PUC-Rio, Programa de Pós-Graduação em Metrologia, 2004.

$$
223 \text { f. : il. ; } 30 \mathrm{~cm}
$$

Dissertação (mestrado) - Pontifícia Universidade Católica do Rio de Janeiro, Programa de Pós-Graduação em Metrologia para a Qualidade Industrial.

Inclui referências bibliográficas

1. Metrologia - Teses. 2. Colorimetria. 3. Padrão virtual. 4. Metameria. I. Hirschler, Robert. II. Frota, Maurício Nogueira. III. Pontifícia Universidade Católica do Rio de Janeiro. Programa de Pós-Graduação em Metrologia para a Qualidade Industrial. IV. Título. 
Dedico este trabalho à Cecília, minha esposa e amiga, e aos meus filhos André, Alice e Alexandre. 


\section{Agradecimentos}

Ao meu orientador Dr. Robert Hirschler pela confiança, orientação, paciência, conhecimentos transmitidos, amizade e parceria para a realização desta jornada acadêmica na área da colorimetria.

Ao meu coordenador e co-orientador, Professor Maurício Nogueira Frota, pelo estímulo e exemplo de entusiasmo no trato de assuntos envolvendo a metrologia, qualidade e inovação.

À PUC-Rio e ao SENAI-CETIQT pela magia da parceria que viabilizou a criação de uma nova área de pesquisa em metrologia da cor, sem a qual este trabalho não seria possível.

À Financiadora de Estudos e Projetos (FINEP) e ao Ministério da Ciência e Tecnologia (MCT) pelo apoio institucional do Fundo Setorial Verde-Amarelo (Convênio FINEP/MCT Convênio FINEP 22.01.0692.00, Referência 1974/01), articulado no contexto de uma cooperação celebrada envolvendo a Sociedade Brasileira de Metrologia, o SENAI/CETIQT e o Programa de Pós-Graduação em Metrologia da PUC-Rio, permitindo enquadrar a presente pesquisa de Mestrado dentre os dez projetospiloto que identificaram como temas de pesquisa de mestrado em metrologia a solução de problemas de interesse industrial.

Aos funcionários da PUC-Rio, especialmente à Eliane Albernaz, dedicada secretária do PósMQI e aos colaboradores do ITUC, pelo apoio ao longo do mestrado.

Aos meus Professores, pelos ensinamentos e dedicação, e aos meus colegas do mestrado, pela integração e apoio fornecido.

Ao amigo Gutemberg pela indicação deste curso e leal parceria nesta caminhada.

Aos meus amigos do CETIQT, Aline, Alexandre, Cássia, Cristiane, Danielle e Liene, pelo inestimável apoio e dedicação.

À colega Jennifer pela oportunidade de ter me apresentado ao Dr. Hirschler para a escolha da proposta de dissertação e apoiado no trabalho.

Aos amigos Fernando Ribeiro e Andre Fernandes, alunos do curso de engenharia industrial têxtil no CETIQT, que dedicaram seu tempo e conhecimentos para a realização de exaustivos experimentos laboratoriais.

À Marinha do Brasil responsável pela minha formação profissional ao longo de mais de trinta anos e a ajuda específica neste trabalho.

À minha esposa, aos meus filhos e à minha mãe pela paciência, compreensão, incentivo e dedicação durante os bons e difíceis momentos do curso.

Aos professores que participaram da Comissão examinadora. 


\section{Resumo}

Régula, Luiz Miguel. Padrões Virtuais e Tolerâncias Colorimétricas no Controle Instrumental das Cores. Rio de Janeiro, 2004. 223p. Dissertação de Mestrado - Programa de Pós-Graduação em Metrologia, Pontifícia Universidade Católica do Rio de Janeiro.

O controle das cores, seja visual ou instrumental, necessita de padrões de comparação. No caso visual esse padrão tem que ser uma amostra real, mas no caso instrumental existe a possibilidade de definir padrões virtuais em forma de valores de refletância espectral. Os padrões reais não são permanentes. A cor muda com o tempo dependendo do substrato, dos corantes ou pigmentos e dependendo da forma de armazenamento essa mudança se torna perceptível após algumas semanas, meses ou anos. Outra limitação dos padrões reais é a dificuldade de reproduzi-los em número suficiente com reprodutibilidade aceitável. Nas indústrias é muito comum usar coleções de cores "padrões" (por exemplo, Pantone, NCS, RAL, ColorCurve), mas mesmo assim sempre há uma diferença entre amostras até da mesma edição, e muito mais entre edições diferentes. Os padrões virtuais não têm essas desvantagens, mas para poder utilizá-los, tem-se que determinar o efeito da estrutura da amostra (não mostrada pelos valores de refletância) na cor percebida, e os limites de tolerância permissíveis na iluminação primária (luz do dia) e em outras iluminações (incandescente, fluorescente). Apresentam-se no texto conceitos relevantes e básicos sobre colorimetria limitados ao escopo abordado no trabalho. O objetivo do trabalho é verificar quantitativamente a diferença instrumental de cor de tecidos com diferentes estruturas, a eficiência de diferentes métodos de cálculo computadorizado de correção de receitas de tingimento e a influência do fenômeno da metameria na tolerância colorimétrica. As amostras têxteis com diferentes estruturas serão tingidas em diversas cores e medidas para a determinação da relação da estrutura com a tolerância instrumental, para vários padrões virtuais e vários iluminantes. Demandou-se a preparação de amostras padrão e medições espectrofotométricas, com elevado controle metrológico, realizadas nas dependências do SENAI-CETIQT, mais especificamente nas UOEQ (Unidade Operacional de Ensaios Químicos) e UOC (Unidade Operacional de Colorimetria). Para a análise dos resultados utilizou-se de métodos estatísticos e matemáticos, auxiliada por planilhas eletrônicas. Complementando a formação acadêmica, o desenvolvimento da pesquisa realizou-se nas 
instalações do SENAI/CETIQT, que possui comprovada competência técnica e científica na área colorimétrica e uma adequada infra-estrutura laboratorial em metrologia da cor que deu suporte ao trabalho.

A presente pesquisa de mestrado voltada ao equacionamento de problemas de interesse industrial desenvolveu-se no contexto de um convênio celebrado entre o Programa de Pós-Graduação em Metrologia da PUC-Rio e o SENAI/CETIQT, que somam esforços para consolidar no País uma nova área de pesquisa em metrologia da cor. Por essa razão, o trabalho foi incluído dentre os 10 projetos-piloto que participaram do Convênio FINEP/MCT no. 22.01.0692.00, Referência 1974/01, que aportou recursos do Fundo Setorial Verde Amarelo para direcionar o esforço de pesquisa em metrologia para a solução de problemas de interesse industrial. Foi com esse propósito que padrões virtuais e tolerâncias colorimétricas no controle instrumental das cores foi selecionado como tema central da pesquisa, cujo desenvolvimento beneficiou-se do ambiente acadêmico e de pesquisa da universidade e da excelente infra-estrutura laboratorial em colorimetria do SENAI/CETIQT. A cooperação inter-institucional estabelecida evidencia o potencial da parceria na solução de complexos problemas de interesse industrial.

\section{Palavras-chave}

Metrologia, colorimetria, padrão virtual, metameria. 


\section{Abstract}

Régula, Luiz Miguel; Hirschler, Robert. Virtual Standards and Colorimetric Tolerances in the Instrumental Control of the Colors. Rio de Janeiro, 2004. 223p. MSc. Dissertation - Programa de PósGraduação em Metrologia, Pontifícia Universidade Católica do Rio de Janeiro.

The control of colors, either visual or instrumental, needs comparison standards. In the visual case this standard has to be a real, physical sample, but in the instrumental case, it is possible to define virtual standards in the form of spectral reflectance values. The real standards are not permanent. Their color changes over time depending on the substrate, the dyes or pigments, and - depending on the storage form - this change becomes perceptible after some weeks, months or years. Another limitation of the real standards is the difficulty to reproduce them in sufficient number with acceptable reproducibility. In industry it is very common to use collections of colors standards (for example, Pantone, NCS, RAL, Color-Curve), but even then there is always a difference between samples even of the same edition, and much more between different editions. Virtual standards do not have these disadvantages, but to be able to use them, the effect of the structure of the sample on the perceived color (not shown by the values of spectral reflectance), as well as the acceptable limits of tolerance under the primary illumination (daylight) and under other illuminations (incandescent, fluorescent) have to be determined. Relevant and basic concepts of colorimetry are presented in the text, limited to the scope of the work. The objective of the work is to quantitatively verify the instrumental color difference between fabrics with different structures, the efficiency of different methods of computerized dye recipe correction calculation and the influence of the phenomenon of metamerism on colorimetric tolerances. Textile samples with different structures will be dyed in diverse colors and the effect of the structure on the instrumental tolerance for some virtual standards and some illuminants will be determined by instrumental measurements. Sample preparation and spectrophotometric measurements were performed under careful metrological control, carried out in the installations of SENAI-CETIQT, more specifically in the UOEQ (Chemical Testing Laboratory) and UOC (Applied Colorimetry Laboratory). Statistical and mathematical methods, assisted by electronic spread sheets, were used for the analysis of the results. Complementing the academic development, the experimental research took place at SENAI/CETIQT, an institution with proven scientific and technical 
capabilities in the field of colorimetry and outstanding laboratorial infrastructure in color metrology, that gave support to the work.

The present M.Sc. Dissertation aiming at the solution of problems of industrial interest was developed in connection with an agreement celebrated between the Post-graduate Programme in Metrology of PUC-Rio and SENAI/CETIQT which cooperate to implement in the country a new area of research in metrology applied to colorimetry. For this reason, the research work was included among the 10 pilot-projects which benefited from the financial support received from the FINEP/MCT Agreement no. 22.01.0692.00, Reference 1974/01 (Green-Yellow governmental Fund) driving the research effort in metrology to the solution of industrial problems. This is why virtual standards and colorimetric tolerances in the instrumental control of the colors was selected as the central topic of this research, which successful development strongly benefited from the academic and scientific environment provided by the university and also by the outstanding laboratory infrastructure in colorimetry offered by SENAI/CETIQT. The agreement established proved to speed up the solution of complex problems requiring advanced knowledge of metrology.

\section{Keywords}

Metrology, colorimetry, virtual standards, metamerism. 


\section{Sumário}

1 Introdução 21

$\begin{array}{lll}1.1 & \text { Objetivos } & 21\end{array}$

1.2 Descrição da Dissertação 22

2 Revisão Bibliográfica 23

2.1 Fundamentos de Colorimetria 23

2.1.1 A Cor 23

2.1.2 Características das Cores 25

$\begin{array}{lll}2.1 .3 & 26\end{array}$

2.1.4 Quantificando a cor no sistema da CIE 28

$\begin{array}{lll}2.1 .4 .1 & \text { Espectrofotômetro } & 28\end{array}$

$\begin{array}{lll}2.1 .5 & \text { Curvas Espectrofotométricas } & 30\end{array}$

2.1.6 Valores Triestímulos X Y Z 35

2.1.7 Repetitividade e exatidão na colorimetria 39

$\begin{array}{lll}\text { 2.1.7.1 A prática de minimizar erros } & 41\end{array}$

2.2 Sistemas de ordenação e classificação de cores 42

2.2.1 Sistemas existentes $\quad 42$

2.2.1.1 O Sistema de Cor Munsell 43

2.2.1.2 Formas de apresentação do Sistema de Cor Munsell 43

2.3 Padrões Reais 46

2.3.1 Coleções 46

$\begin{array}{lll}2.3 .2 & \text { Azulejos } & 48\end{array}$

2.3.2.1 Desenvolvimento dos Padrões de Cor 48

2.3.2.2 Padrões de cor cerâmica (Série I) 48

2.3.2.3 Padrões de cor cerâmica (Série II) 49

2.3.2.4 Padrões neutros 50

2.3.2.5 Calibração 51

2.3.2.6 Medições dos Padrões 51

2.3.2.7 Termocromismo 51

2.3.2.8 Uso \& aplicações 52

2.4 Padrões Virtuais $\quad 53$ 
$\begin{array}{lll}2.5 & \text { Metameria } & 60\end{array}$

2.5.1 A causa da Metameria 63

2.5.2 A definição de metameria 66

$\begin{array}{lll}2.5 .3 & \text { Metameria na prática } & 68\end{array}$

$\begin{array}{lll}\text { 2.5.4 Grau de metameria } & 70\end{array}$

2.5.4.1 Índice geral de metameria 70

2.5.4.2 Índice especial de metameria CIE $\quad 71$

2.5.4.2.1 Índice Especial de Metameria para Mudança de lluminante 73

2.5.4.2.2 Índice Especial de Metameria para Mudança de Observador 75

2.5.4.3 Redução metameria \& custo / formulação computadorizada 76

$\begin{array}{lll}2.6 & \text { Cor e Estrutura } & 78\end{array}$

2.6.1 Refletância, absorvância e dispersão - o modelo simplificado do

Kubelka e Munk $\quad 78$

$\begin{array}{lll}\text { 2.6.2 Título da fibra e do fio } & 79\end{array}$

2.6.3 Padronagem do tecido 80

2.6.4 O efeito da separação dos fios (cobertura) 82

2.6.5 Brilho 84

3 Exemplo de Padronização de Cores $\quad 89$

3.1 Normas envolvendo a padronização das cores 89

4 Experimental $\quad 91$

4.1 Matização Computacional e Receitas Metaméricas 91

4.1.1 Materiais 93

4.1.1.1 Substratos 93

4.1.1.2 Corante 94

$\begin{array}{lll}\text { 4.1.1.3 } & \text { Auxiliares } & 94\end{array}$

4.1.1.4 Água 94

$\begin{array}{lll}\text { 4.1.2 Equipamentos } & 95\end{array}$

4.1.2.1 Balança 95

4.1.2.2 Dosimat 96

4.1.2.3 Pipeta Eletrônica 96

4.1.2.4 Aparelho de Tingimento 96

$\begin{array}{lll}\text { 4.1.2.5 } & \text { Espectrofotômetro } & 97\end{array}$

$\begin{array}{lll}4.1 .3 & \text { Tingimentos } & 97\end{array}$

$\begin{array}{lll}\text { 4.1.3.1 } & \text { Processo } & 98\end{array}$

$\begin{array}{lll}\text { 4.1.3.2 } & \text { Receitas } & 99\end{array}$ 
4.1.4 Medição da Cor 101

$\begin{array}{lll}\text { 4.1.4.1 Uniformidade } & 102\end{array}$

$\begin{array}{lll}\text { 4.1.4.2 Repetitividade } & 103\end{array}$

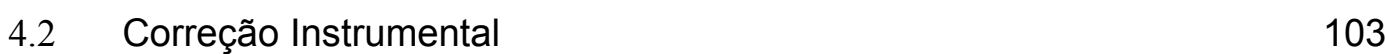

4.2.1 Determinação da matriz de influência com tingimentos com corantes $\begin{array}{ll}\text { individuais } & 106\end{array}$

4.2.2 Determinação da matriz de influência com tingimentos com combinações 108

$\begin{array}{lll}\text { 4.2.3 Cálculo das receitas com correção } & 109\end{array}$

5 Resultados e Discussão 111

$\begin{array}{ll}5.1 & 111\end{array}$

$\begin{array}{lll}5.2 & \text { Repetitividade } & 111\end{array}$

5.3 Correção Instrumental 112

$\begin{array}{lll}\text { 5.3.1 Algodão X Tencel } & 114\end{array}$

$\begin{array}{lll}\text { 5.3.2 Algodão x Gorgurão } & 115\end{array}$

$\begin{array}{ll}\text { 5.3.3 Gorgurão x Tencel } & 117\end{array}$

5.3.4 Tafetá x Gorgurão \& Tafetá x Sarja 119

5.4 Efeito da Estrutura na cor dos tecidos 122

5.4.1 Cor Padrão 1122

5.4.2 Cor Padrão 2

5.4.3 Cor Padrão $3 \quad 126$

5.4.4 Diferença de cor entre Sarja x Tafetá, Tafetá e Gorgurão e entre Tafetá 40 e $50 \quad 128$

$\begin{array}{lll}5.5 & \text { Seleção de Padrões } & 130\end{array}$

5.6 Efeito da Metameria na Prática 133

5.7 Aplicação da Tolerância Colorimétrica 135

6 Conclusões e Recomendações 139

7 Referências Bibliográficas 143 


\section{Lista de figuras}

Figura 1 - Espectro visível 23

Figura 2 - Cor: os três componentes: fonte, objeto e observador (Hunter,1987)24

Figura 3 - A cor apresentada esquematicamente: curvas espectrais 24

Figura 4 - Características das cores (Hirschler, 2002) 26

Figura 5 - Comparação entre uma Fonte (2854K) e um lluminante CIE (A): as curvas ficam sobrepostas (Hirschler, 2002) 27

Figura 6 - Comparação entre uma Fonte (6500K) e um lluminante CIE (D65) (Hirschler, 2002) 27

Figura 7 - Espectrofotômetro (Berms, 2000) 29

Figura 8 - Geometria de medição (Minolta, 1986) 30

Figuras 9 - Exemplo de curvas espectrais $\quad 31$

Figura 10 - Refletância e brilho (Hunter, 1987) 32

Figura 11 - Experimento de Grassmann (Hunter, 1987) 33

Figura 12 - Funções de matização de cores (Dados da norma CIE/ISO) 34

Figura 13 - A cor apresentada esquematicamente: valores triestímulos $\quad 34$

Figura 14 - Espaço CIELAB; espaço psicométrico 36

Figura 15 - Espaço CIELAB (Minolta, 1986) 36

Figura 16 - Espaço CIELAB; significado geométrico das coordenadas (Hirschler, 2000) 36

Figura 17 - Diferença de cor no espaço CIELAB 38

Figura 18 - Árvore de cor Munsell (www.gretagmacbeth.com) 43

Figura 19 - Sistema ordenação de cores Munsell (Software CMC da GretagMacbeth, www.gretagmacbeth.com) 44

Figura 20 - Coleção PANTONE (Hirschler, 2002) 47

Figura 21 - Notação da Coleção PANTONE (Hirschler, 2002) 47

Figura 22 - Padrões de cerâmica série I (CERAM, 2003) 49

Figura 23 - Padrões de cerâmica série CCSII (CERAM, 2003) 49

Figura 24 - Padrões cerâmicos neutros (CERAM, 2003) 50

Figura 25 - Processo de aprovação de cores $\quad 58$

Figura 26 - Constância de uma Cor $\quad 61$

Figura 27 - Constância de cores $\quad 61$

Figura 28 - Inconstância da cor $\quad 62$

Figura 29 - Cores iguais $\quad 62$ 
Figura 30 - Cores diferentes $\quad 63$

Figura 31 - Cores metaméricas $\quad 63$

Figura 32 - Fatores de peso; D65/10 (Dados da norma CIE/ISO) 64

Figura 33 - Fatores de peso; A/10 (Dados da norma CIE/ISO) 64

Figura 34 - Fluxograma de predição (Hirschler, 2002) 66

$\begin{array}{ll}\text { Figura } 35 \text { - Metameria } & 67\end{array}$

Figura 36 - Exemplo de curvas de pares metaméricos 72

Figura 37 - O significado do modelo Kubelka e Munk (Kuehni, 1975) 78

Figura 38 - O efeito do título (diâmetro) da fibra na concentração aparente do corante $\quad 80$

Figura 39 - Modelo de refletância de um substrato têxtil (Lee \& Sato, 2001) 81

Figura 40 - Orientação dos fios no tecido (Lambert et al., 1986) 81

Figura 41 - Estrutura de tecidos $\quad 82$

Figura 42 - Representação do modelo simplificado de Goldfinger e Paige (1977)

Figura 43 - A refletância $R_{F R}$ do tecido com densidade de fios FR 83

Figura 44 - Curvas de refletância de materiais usados na pesquisa de Kurz e Troska (1970) 84

Figura 45 - A distribuição espacial da refletância em vários substratos com ângulo de iluminação de 45 graus (Kurz e Strocka, 1970) 85

Figura 46 - Esquema da visão microscópica da incidência da luz em um objeto $\begin{array}{ll}\text { (Hunter, 1987) } & 87\end{array}$

Figura 47 - Curvas espectrofotométricas de cores padrão 91

Figura 48 - Balança Analítica 95

Figura 49 - Dosimat 96

Figura 50 - Pipeta Eletrônica $\quad 96$

$\begin{array}{ll}\text { Figura } 51 \text { - Vista externa e interna do Tubotest } & 97\end{array}$

Figura 52 - Espectrofotômetros 3600d e ColorView $9000 \quad 97$

Figura 53 - Amostras preparadas para serem tintas 98

Figura 54 - Representação gráfica da medida de uniformidade 102

Figura 55 - Diferenças entre as coordenadas colorimétricas da cor Padrão 1

(Tencel e Algodão ), antes e após as correções 115

Figura 56 - Diferenças entre as coordenadas colorimétricas da cor Padrão 2

(Tencel e Algodão ), antes e após as correções 115

Figura 57 - Diferenças entre as coordenadas colorimétricas da cor Padrão 3

(Tencel e Algodão ), antes e após as correções 115 
Figura 58 - Diferenças entre as coordenadas colorimétricas dos substratos

Algodão e Gorgurão para a cor Padrão 1, antes e após as correções 117

Figura 59 - Diferenças entre as coordenadas colorimétricas dos substratos

Algodão e Gorgurão para a cor Padrão 2, antes e após as correções 117

Figura 60 - Diferenças entre as coordenadas colorimétricas dos substratos

Algodão e Gorgurão para a cor Padrão 3, antes e após as correções 117

Figura 61 - Diferenças entre as coordenadas colorimétricas dos substratos

Gorgurão e Tencel para a cor Padrão 1

Figura 62 - Diferenças entre as coordenadas colorimétricas dos substratos

Gorgurão e Tencel para a cor Padrão 2, antes e após a correção, respectivamente

Figura 63 - Diferenças entre as coordenadas colorimétricas dos substratos

Gorgurão e Tencel para a cor Padrão 3, antes e após a correção respectivamente

Figura 64 - Diferenças entre as coordenadas colorimétricas dos substratos

Tafetá x Gorgurão para a cor Padrão Cinza Escuro, antes e após a correção respectivamente

Figura 65 - Diferenças entre as coordenadas colorimétricas dos substratos

Tafetá x Sarja para a cor Padrão Cinza Escuro, antes e após a correção respectivamente

Figura 66 - Diferenças entre as coordenadas colorimétricas dos substratos Tafetá x Gorgurão para a cor Padrão Cinza Claro, antes e após a correção respectivamente

Figura 67 - Diferenças entre as coordenadas colorimétricas dos substratos Tafetá x Sarja para a cor Padrão Cinza Claro, antes e após a correção respectivamente

Figura 68 - Diferenças entre as coordenadas colorimétricas dos substratos

Tafetá x Gorgurão para a cor Padrão Bege, antes e após a correção respectivamente

Figura 69 - Diferenças entre as coordenadas colorimétricas dos substratos Tafetá x Sarja para a cor Padrão Bege, antes e após a correção respectivamente

122

Figura 70 - Diferenças entre as coordenadas colorimétricas dos artigos Tencel e Algodão (SIN) e (SEX)

Figura 71 - Diferenças entre as coordenadas colorimétricas dos artigos Tencel e Algodão (45/0) 
Figura 72 - Diferenças entre as coordenadas colorimétricas dos artigos Tencel e Gorgurão (SIN) e (SEX)

Figura 73 - Diferenças entre as coordenadas colorimétricas dos artigos Tencel e Gorgurão (45/0)

Figura 74 - Diferenças entre as coordenadas colorimétricas dos artigos Algodão e Gorgurão (SIN) e (SEX) 124

Figura 75 - Diferenças entre as coordenadas colorimétricas, dos artigos Algodão e Gorgurão (45/0)

Figura 76 - Diferenças entre as coordenadas colorimétricas dos artigos Tencel e Gorgurão (SIN) e (SEX) 125

Figura 77 - Diferenças entre as coordenadas colorimétricas dos artigos Tencel e Algodão (45/0) 125

Figura 78 - Diferenças entre as coordenadas colorimétricas dos artigos Tencel e Gorgurão (SIN) e (SEX) 125

Figura 79 - Diferenças entre as coordenadas colorimétricas dos artigos Tencel e Gorgurão (45/0)

Figura 80 - Diferenças entre as coordenadas colorimétricas dos artigos Gorgurão e Algodão (SIN) e (SEX)

Figura 81 - Diferenças entre as coordenadas colorimétricas dos artigos Gorgurão e Algodão (45/0) 126

Figura 82 - Diferenças entre as coordenadas colorimétricas dos artigos Tencel e Algodão (SIN) e (SEX) 126

Figura 83 - Diferenças entre as coordenadas colorimétricas dos artigos Tencel e algodão (45/0)

127

Figura 84 - Diferenças entre as coordenadas colorimétricas dos artigos Tencel e Gorgurão (SIN) e (SEX)

Figura 85 - Diferenças entre as coordenadas colorimétricas dos artigos Tencel e Gorgurão (45/0)

Figura 86 - Diferenças entre as coordenadas colorimétricas dos os artigos Gorgurão e Algodão (SIN) e (SEX)

Figura 87 - Diferenças entre as coordenadas colorimétricas dos artigos Gorgurão e Algodão (45/0)

128

Figura 88 - Verificação de ( $\Delta \mathrm{L}^{*}, \Delta \mathrm{a}^{*}, \Delta \mathrm{b}^{*}, \Delta \mathrm{C},{ }^{*} \Delta \mathrm{H}$ e $\left.\Delta \mathrm{E}\right)$ para os artigos de Sarja e Tafetá

Figura 89 - Verificação gráfica de $\left(\Delta \mathrm{L}^{*}, \Delta \mathrm{a}^{*}, \Delta \mathrm{b}^{*}, \Delta \mathrm{C},{ }^{*} \Delta \mathrm{H}\right.$ e $\left.\Delta \mathrm{E}\right)$ para os artigos de Tafetá 
Figura 90 - Verificação gráfica de $\left(\Delta \mathrm{L}^{*}, \Delta \mathrm{a}^{*}, \Delta \mathrm{b}^{*}, \Delta \mathrm{C},{ }^{*} \Delta \mathrm{H}\right.$ e $\left.\Delta \mathrm{E}\right)$ para os artigos de Tafetá e Gorgurão

Figura 91 - Utilização do Padrão Virtual

Figura 92 - Curvas metaméricas de amostras matizadas 


\section{Lista de tabelas}

Tabela 1 - Diferenças entre cores metaméricas $\quad 72$

Tabela 2 - Tabela de Cores de Padrão Real 90

Tabela 3 - Pontos da curva de refletância dos padrões Bege, Cinza escuro e Cinza claro $\quad 92$

Tabela 4 - Diferentes tipos de artigos $\quad 93$

Tabela 5 - Parâmetros usados para verificação da qualidade da água a ser utilizada no tingimento $\quad 95$

Tabela 6 - Concentração dos corantes para as cores padrões 99

Tabela 7 - Concentração dos corantes para as cores metaméricas 99

Tabela 8 - Concentração dos corantes da cor Padrão 1 e suas variações em + $20 \% \quad 100$

Tabela 9 - Concentração dos corantes da cor Padrão 2 e suas variações em + $20 \% \quad 100$

Tabela 10 - Concentração dos corantes da cor Padrão 3 e suas variações em + $20 \%$

Tabela 11 - Receita para tingimentos com os corantes individuais e para as cores padrões 1,2 e $3 \quad 100$

Tabela 12 - Receita para tingimentos com os corantes individuais e para as cores padrões 1,2 e $3 \quad 100$

Tabela 13 - Exemplo de receitas para calcular a correção na base de tingimentos com combinações 109

Tabela 14 - Exemplo da correção das receitas 110

Tabela 15 - Resultados da Uniformidade de 3 partidas $\Delta \mathrm{E}^{*}{ }_{\text {uni }} \quad 111$

Tabela 16 - Resultados da Repetitividade entre os canecos das 3 partidas realizadas 112

Tabela 17 - Concentração dos corantes a partir dos cálculos de correção pelo método de correções com combinações, representando as novas receitas para as cores 1,2 e 3

Tabela 18 - Concentração dos corantes a partir dos cálculos de correção pelo método de correções com tingimentos individuais, representando as novas receitas para as cores 1,2 e 3

Tabela 19 - Concentração dos corantes a partir dos cálculos de correção pelo método de correções com tingimentos individuais, representando as novas 
receitas para as cores cinza escuro, cinza claro e bege

Tabela 20 - Valores das coordenadas colorimétricas e diferença de cor, medidos em espectrofotômetro, para os artigos de Tencel e Algodão nas Cores 1, 2 e 3, antes e depois das correções do Algodão

Tabela 21 - Valores das coordenadas colorimétricas e diferença de cor, medidos em espectrofotômetro, para os artigos de Algodão e Gorgurão nas Cores 1, 2 e 3, antes e depois das correções

Tabela 22 - Valores das coordenadas colorimétricas e diferença de cor, medidos em espectrofotômetro, para os artigos de Gorgurão e Tencel nas Cores 1, 2 e 3, antes e depois da correção

Tabela 23 - Valores das coordenadas colorimétricas e diferença de cor , medidos nem espectrofotômetros, para os artigos Tafetá, Gorgurão e Sarja nas cores cinza escuro, cinza claro e bege, antes e após a correção realizada pelo método 2

Tabela 24 - Medição da diferença existente entre três tecidos da tabela 4 com três geometrias $\Delta \mathrm{E}_{\mathrm{CIELAB}}$ Cor 1

122

Tabela 25 - Medição da diferença existente entre três tecidos com três geometrias $\triangle \mathrm{E}_{\mathrm{CIELAB}}$ Cor $2 \quad 124$

Tabela 26 - Medição da diferença existente entre três tecidos com três geometrias $\triangle \mathrm{E}_{\text {CIELAB }}$ Cor $3 \quad 126$

Tabela 27 - Medição da diferença das coordenadas colorimétricas dos tecidos Sarja e Tafetá ambos título 40 - Cor 2

Tabela 28 - Medição da diferença das coordenadas colorimétricas dos tecidos de Tafetá títulos 40 e 50 - Cor 2

Tabela 29 - Medição da diferença das coordenadas colorimétricas dos tecidos de Tafetá e Gorgurão - Cor Cinza Escuro

Tabela 30 - Uma formatação de norma colorimétrica 132

Tabela 31 - Confrontação de normas de cores 136

Tabela 32 - Diferenças de cor pela fórmula CMC2:1 136

Tabela 33 - Diferenças de coordenadas colorimétricas entre amostras 


\section{Apêndices e anexos}

$\begin{array}{ll}\text { Apêndice A - K/S unitário } & 148\end{array}$

Apêndice B - Refletância corantes $\quad 167$

Apêndice C - Refletância calculada tricomia 170

Apêndice D - Refletância medida corantes 176

Apêndice E - Gráficos comparativos correções individuais 179

Apêndice F - Diferenças de coordenadas colorimétricas 185

Apêndice G - Cálculo da Refletância das cores das amostras matizadas 191

Apêndice $\mathrm{H}$ - Matriz de correção e formulação da nova receita 213

Apêndice I - Amostras do Padrão Visual, Padrão Tafetá matizado e Gorgurão e

$\begin{array}{ll}\text { Sarja tintos } & 219\end{array}$

Apêndice $\mathrm{J}$ - Amostras do Método de correção - Tingimentos Individuais no

$\begin{array}{ll}\text { Gorgurão } & 220\end{array}$

Apêndice K - Amostras dos Resultados do Método de Correção com

Tingimentos Individuais no Gorgurão 221

Apêndice $L$ - Amostras do Método de Correção - Tingimentos Individuais na

Sarja 222

Apêndice $M$ - Amostras dos Resultados do Método de Correção com

$\begin{array}{ll}\text { Tingimentos Individuais na Sarja } & 223\end{array}$

\section{Lista de Quadros}

Quadro 1 - Cores padrão matizadas para os experimentos

Quadro 2 - Diferenças entre os valores da norma (padrões virtuais) e as amostras têxteis (padrões físicos) no substrato tafetá 92

Quadro 3 - Tingimentos com corantes individuais 106

Quadro 4 - Índice de metameria com correção multiplicativa de amostras matizadas 